\title{
Nell-1 induces acrania-like cranioskeletal deformities during mouse embryonic development
}

\author{
Xinli Zhang ${ }^{1}$, Catherine M Cowan ${ }^{2}$, Xinquan Jiang ${ }^{3}$, Chia Soo ${ }^{4}$, Steve Miao ${ }^{1,5}$, \\ Dale Carpenter ${ }^{6}$, Benjamin $\mathrm{Wu}^{2, *}$, Shun'ichi Kuroda ${ }^{7,8, *}$ and Kang Ting ${ }^{1,5, *}$
}

${ }^{1}$ Dental and Craniofacial Research Institute, University of California, Los Angeles, CA, USA; ${ }^{2}$ Department of Bioengineering, University of California, Los Angeles, CA, USA; ${ }^{3}$ Oral Bioengineering Laboratory, Shanghai Research Institute of Stomatology, Shanghai Second Medical University, Shanghai, China; ${ }^{4}$ Division of Plastic and Reconstructive Surgery, Department of Surgery, University of Southern California, Los Angeles, CA, USA; ${ }^{5}$ Section of Orthodontics, School of Dentistry, University of California, Los Angeles, CA, USA; ${ }^{6}$ Department of Ophthalmology, University of California, Irvine, CA, USA; ${ }^{7}$ Research Center, Kobe University, Kobe, Japan and ${ }^{8}$ Department of Structural Molecular Biology, Institute of Scientific and Industrial Research, Osaka University, Ibaraki, Osaka, Japan

\begin{abstract}
We previously reported NELL-1 as a novel molecule overexpressed during premature cranial suture closure in patients with craniosynostosis (CS). Nell-1 overexpression also results in premature suture closure/ craniosynostosis in newborn transgenic mice. On a cellular level, increased levels of Nell-1 induce osteoblast differentiation and apoptosis. In this report, mice over-expressing Nell-1 were examined during embryonic development as well as shortly after birth for further analysis of craniofacial defects including neural tube defects (NTDs). The results demonstrated that overexpression of Nell-1 could induce acrania at relatively late gestation stage (E15.5) in mouse embryos, through massive apoptosis in calvarial osteoblasts and neural cells. The induced apoptosis was associated with an increase in Fas and Fas-L production. In addition, transgenic E15.5 and newborn transgenic mice with the CS phenotype displayed distortion of the chondrocranium associated with premature hypertrophy and increased apoptosis of chondrocytes. These findings were also verified in vitro with primary chondrocytes transduced with AdNell-1. In conclusion, Nell-1 overexpression can induce craniofacial anomalies associated with neural tube defects during embryonic development and may involve mechanisms of massive apoptosis associated with the Fas/Fas-L signaling pathway. NELL-1: used when describing the human gene; NELL-1: used when describing the human protein; Nell-1: used when describing the rodent gene; Nell-1: used when describing the rodent protein Laboratory Investigation (2006) 86, 633-644. doi:10.1038/labinvest.3700430; published online 1 May 2006
\end{abstract}

Keywords: apoptosis; acrania; Nell-1; transgenic

Acrania is defined as partial or complete absence of flat bones of the cranial vault with complete, but abnormal development of the cerebral hemisphere. ${ }^{1}$ It has been controversial to use the term acrania as a synonym of acalvaria, which is one type of neural tube defect (NTD). ${ }^{2}$ Previously, NTDs were divided into two types, open (neuralation defects) and closed (postneurulation defects), by Lemire. ${ }^{3}$ The

Correspondence: Dr K Ting, DMD, DMEDSC, Dental and Craniofacial Research Institute, University of California, 10833 Le Conte Avenue, CHS 30-117, Los Angeles, CA 90095, USA.

E-mail: kting@ucla.edu

${ }^{*}$ Co-senior authors.

Received 3 November 2005; revised 16 March 2006; accepted 21 March 2006; published online 1 May 2006 open NTDs such as anencephaly and myelomeningocele occur prior to closure of the neural tube in earlier embryonic stages, while the closed ones including acalvaria, encephalocele, and lipomeningocele arise after closure of the neural tube. ${ }^{3}$

In humans, anencephaly, characterized as brain absent above the orbit with bulging eyes, ${ }^{1}$ represents one of the most common NTDs and occurs in 0.1$0.9 \%$ of all births. ${ }^{2}$ Fusion of the neural tube requires coordination of several biological processes including cell migration, proliferation, and apoptosis. ${ }^{4}$ More than 80 mouse mutants display NTDs ${ }^{5}$ and at least 50 single gene mutations have been reported to cause NTDs. ${ }^{6}$ Although the exact etiologies of these defects are not completely known, both genetic and environmental factors have 
been implicated. ${ }^{4}$ Acalvaria is a rare congenital postneurulation defect classified etiologically as primary and secondary. Primary acalvaria is distinguished by a complete absence of calvarial bones, dura mater, and associated muscles in the presence of the cerebral hemisphere, normal cranial skin, normal skull base, and normal facial bones. ${ }^{7}$ It was postulated that the failure of differentiation of specific portions of the cephalic neural crest or the failure of mesodermal migration deep within the ectoderm might be involved in this development. ${ }^{8-10}$ Secondary acalvaria was usually seen with amniotic bands syndrome and anencephaly or its precursor exencephaly. It is obvious that primary acalvaria differs from acrania by definition, but acrania is also associated with anencephaly or exencephaly. ${ }^{11}$ Moreover, it is clinically important to differentiate acalvaria from acrania in utero by sonography as the brain can be normal and potentially treatable in the former, but the latter usually progresses to anencephaly. ${ }^{11}$

During early embryogenesis, pluripotent cranial neural crest (CNC) cells arise from the lateral edges of the neural folds and migrate through the lateral ridges of the neural plate. ${ }^{12-14}$ Eventually, CNC cells become mesenchymal cells that form the components of the craniofacial structures, including the orofacial prominences and mandibular arch..$^{15,16}$ Unlike trunk neural crest cells, CNC cells differentiate into both cartilage and bone. ${ }^{17}$ Alterations in CNC cell fate are associated with craniofacial deformities, such as Treacher Collins syndrome. ${ }^{18}$ In this syndrome, patients have a Tcof1 loss of function mutation that induces massive apoptosis in the neuroepithelium of the cranial neural folds and the neural tube. ${ }^{19}$ Owing to species-specific differences in craniofacial development, Tcof1 ${ }^{+/-}$mice exhibit exencephaly and craniofacial skeletal deficiencies. ${ }^{19}$ Furthermore, in vitro and in vivo studies have demonstrated that signaling through the CD95/ Fas pathway resulted in increases apoptosis in human and murine osteoblasts. ${ }^{20-22}$

We previously reported the isolation and identification of a novel gene, $N E L L-1$, in human unilateral coronal craniosynostosis (CS) ${ }^{23}$ (concurrently identified by Watanabe et $\mathrm{l}^{24}$ ). NELL-1 is a secretory protein containing 810 amino acids. ${ }^{25,26}$ NELL-1 encodes a secretory signal peptide sequence, an $\mathrm{NH}_{2}$-terminal thrombospondin (TSP)-like module, five von Willebrand factor $\mathrm{C}$ domains, and six EGFlike domains. ${ }^{27} \mathrm{NELL-1}$ expression is upregulated in fused or prematurely fusing coronal sutures as compared to wild-type coronal sutures. The NELL1 gene is preferentially expressed in neural crest derived tissues including intramembranous cranial bone and neural tissue. ${ }^{23}$ Furthermore, our Nell-1 transgenic mouse model demonstrates craniosynostosis phenotypes of varied degrees and increased apoptosis in osteoblasts. ${ }^{28}$ Also, AdNell-1 transduced MC3T3-E1 osteoblasts undergoing apoptosis demonstrate increased Fas expression. ${ }^{22}$
Although the precise molecular mechanism of Nell-1 is not well understood, the current study further examines the role of Nell-1 in the development of NTDs of Nell-1 transgenic mice, since we have observed an exencephaly-like phenotype during embryogenesis. ${ }^{28}$ We have further confirmed that acrania-like cranioskeletal deformities occur among E15.5 Nell-1 transgenic mouse embryos by examining more litters and different gestational stages. In addition to severe CS, several newborn transgenic mice displayed encephaloceles characterized by occipital bone defects with brain herniation. Evidence of increased Fas signaling in apoptosis of other tissues, both neural and cartilaginous, has been detected, ${ }^{29-31}$ but has not yet been associated with craniofacial deformities. In addition, the similar spatiotemporal expression pattern of both Nell-1 and Fas/Fas-L, along with the occurrence of apoptosis in transgenic mouse embryos, indicated a possible mechanism of Nell-1 induced apoptosis in mouse craniofacial defects.

\section{Materials and methods}

\section{Transgenic Newborn and Embryonic Mice with Overexpressing Nell-1}

Transgenic mice overexpressing Nell-1 were constructed and genotyped by PCR as reported previously. ${ }^{28}$ Three litters of newborn mice from F1 transgenic mice mated with wild-type littermates were examined for NTDs. The breeding strategy for mouse embryos at different stages in this study was set timed-mating between heterozygous female transgenic mice with age-matched male wild-type mice. The presence of a vaginal plug was defined as $0.5 \mathrm{dpc}$ or gestational day 0.5 (E0.5). Two litters of E12.5 and four litters of E15.5 embryos were collected and genotypes were confirmed by PCR as previously described. ${ }^{28}$ Pregnant mice were anesthetized and whole embryos were removed and fixed immediately in $4 \%$ paraformaldehyde. Hematoxylin and eosin (H\&E) staining was performed on paraffin embedded tissues using standard protocols. ${ }^{28}$

\section{Immunohistochemistry}

Paraffin embedded sections were incubated with anti-Nell-1 antibody, ${ }^{26}$ anti-Fas antigen, or anti-Fasligand antibodies (Santa Cruz Biotechnology, Santa Cruz, CA, USA) at a 1:100 dilution. ABC complex (Vector Laboratories, Burlingame, CA, USA) was applied on the sections following the incubation with biotinylated secondary antibody (Dako Corporation, Carpinteria, CA, USA). DAB in brown color or AEC plus substrate in red color (Dako Corporation) was used as a chromagen. The sections were counterstained with light Hematoxylin. The negative control with PBS substituted for primary antibody was run along side of each reaction. 


\section{TdT-Mediated dUTP Nick End Labeling}

Paraffin embedded tissue sections or chondrocyte cell cultures were labeled with DeadEnd Colorimetric Apoptosis Detection System (Promega Corp., Madison, WI, USA) as previous published. ${ }^{32}$ Briefly, the paraffin embedded tissue sections or cell cultures were treated with proteinase $\mathrm{K}$ and labeled with $\mathrm{TdT}$ reaction mix containing equilibration buffer, biotinylated nucleotide mix and TdT enzyme. The negative control was carried out identically to the experimental labeling with the exception of TdT enzyme. Streptavidin-HRP and DAB (Dako Corporation) substrates were used for visualization.

\section{Overexpression of Nell-1 in Primary Chondrocytes}

Goat auricular cartilage was minced to $1 \mathrm{~mm}^{3}$ pieces and digested with $0.25 \%$ trypsin/ $1 \mathrm{mM}$ EDTA at room temperature for $30 \mathrm{~min}$, followed by $3 \mathrm{mg} / \mathrm{ml}$ collagenase II (Sigma, St Louis, MO, USA) digestion with shaking at $37^{\circ} \mathrm{C}$ for $6 \mathrm{~h}$. The cell suspension was filtered through a $70 \mu \mathrm{m}$ strainer and the chondrocytes were then pelleted by centrifugation. After washing with PBS, the cells were cultured in DMEM (Gibco BRL, Grand Island, NY, USA) plus $10 \%$ fetal calf serum (Hyclone, Logan, UT, USA), $100 \mathrm{U} / \mathrm{ml}$ penicillin and $100 \mathrm{mg} / \mathrm{l}$ streptomycin at $37^{\circ} \mathrm{C}$ with $5 \% \mathrm{CO}_{2}$. Second passage cells were used for gene transfer studies. Briefly, $1.8 \times 10^{5}$ chondrocytes were seeded on a six-well plate 1 day before gene transfer. An adenovirus containing the Nell-1 full length cDNA coding sequence (AdNell-1) was constructed and used as previously described for gene transfer. ${ }^{28}$ Chondrocytes were transduced with 50 and $200 \mathrm{pfu} / \mathrm{cell}$ for $1 \mathrm{~h}$ using AdNell-1 or AdLacZ for control. The culture medium was changed to DMEM containing $2 \mathrm{mmol} / \mathrm{l}$ L-glutamine, $10^{-8} \mathrm{~mol} / \mathrm{l}$ dexamethasone, $50 \mu \mathrm{g} / \mathrm{ml}$ ascorbic acid, $10 \mathrm{mmol} / \mathrm{l} \beta$-glycerophosphate (Sigma) 3 days posttransduction. Flow cytometry and TdT-mediated dUTP nick end labeling (TUNEL) analysis were performed 6 and 9 days after gene transfection, respectively. Proteins from additional cells were harvested in cell lysis buffer using standard protocols and used for Western blot analysis with antiNell- ${ }^{26}$ and anti- $\beta$-actin antibodies (Sigma).

\section{Flow Cytometry Analysis of Apoptosis in Chondrocytes with Annexin V and PI Staining}

AdNell-1 or AdLacZ (each 50 pfu/cell) transduced chondrocytes were washed with PBS and then trypsinized. Cell pellets were resuspended in $1 \times$ binding buffer containing $0.01 \mathrm{M}$ HEPES/NaOH, pH7.4, $140 \mathrm{mM} \mathrm{NaCl}$, and $2.5 \mathrm{mM} \mathrm{CaCl}_{2}$ at a concentration of $1 \times 10^{6}$ cells $/ \mathrm{ml}$. For flow cytometry, $1 \times 10^{5}$ cells were stained with annexin $\mathrm{V}$ and propidium iodide (PI) (BD Biosciences, Palo
Alto, CA, USA) for $15 \mathrm{~min}$ at room temperature in the dark. The flow cytometry analysis was performed within an hour of staining. The $\chi^{2}$ test was used to analyze the selected raw data as a measure of agreement between observed and expected frequencies for profile selections. An a priori alpha was set at $(P \leq 0.05)$. Biological experiments were repeated three times.

\section{Results}

\section{Acrania in Nonviable E15.5 Nell-1 Overexpression Mice}

The percentage of positive transgenic mice was $47.06 \%$ (8/17) for E12.5 and 44.44\% (16/36) for E15.5 embryos. The relatively lower transgenic rate than predicted $(50 \%)$ may be due to early miscarriages of some embryos. Viable E12.5 and E15.5 fetuses were phenotypically indistinguishable among transgenic and wild-type littermates. Among E15.5 progeny, 16 Nell-1 transgenic positive mice were detected with thirteen viable and three nonviable embryos (18.75\%) showing exencephalic-like phenotypes (Figure 1a). Calvarial bone defects in the foramina displayed protruding brain tissue. Histologically, most of the cerebral tissues within the cranial cavity were partially covered with dura mater. There is no scalp and skin overlying the protruding brain mass, but the chondrocranial base was in intact (Figure $1 \mathrm{~b}$ and $\mathrm{c}$ ). The histological characteristics of the abnormal embryos and their occurrence only at relatively late embryonic stage of E15.5 fit the definition of a rare human congenital disease called acrania which is often associated with exencephaly. ${ }^{1}$ All positive transgenic mice contained approximately 50 copies of the Nell-1 transgene as compared to standards of gene copy, ${ }^{28}$ but their expression levels were highly variable among individuals as detected by immunohistochemistry (data not shown). A significant upregulation of Nell-1 production was distributed in all cells of brain and dura mater in transgenic mice with acrania, while Nell-1 was produced only in restricted areas of wild-type mouse tissues (Figure 1d). Furthermore, in situ TUNEL demonstrated drastically increased apoptosis in several areas including the protruding brain mass and dura mater (Figure 1e and $\mathrm{f}$ ).

\section{Fas and Fas-L Production in E15.5 and E12.5 Nell-1 Overexpression Mice}

Previously, microarray analysis of MC3T3-E1 osteoblasts overexpressing Nell-1 reported an upregulation of genes associated with cell death, most notably Fas. $^{22}$ Thus, the expression of Fas and Fas-L were investigated in E15.5 mice, as these mice displayed early evidence of the phenotype. The data demonstrated markedly increased Fas receptor 


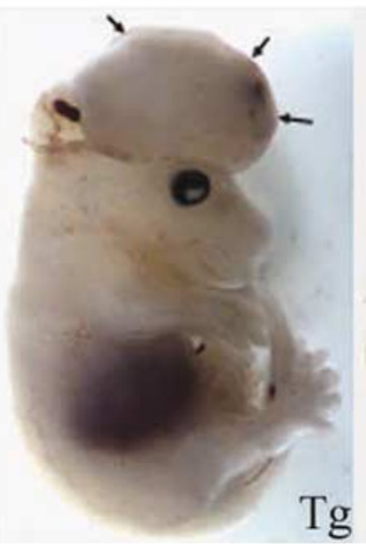

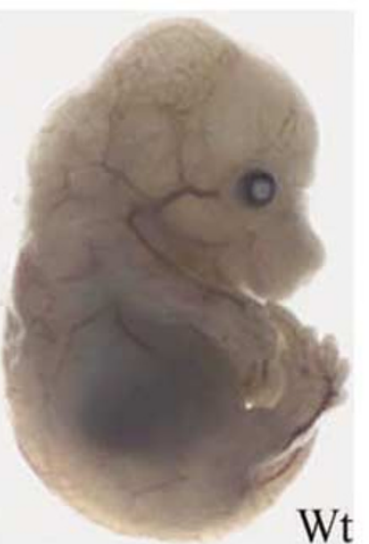

b
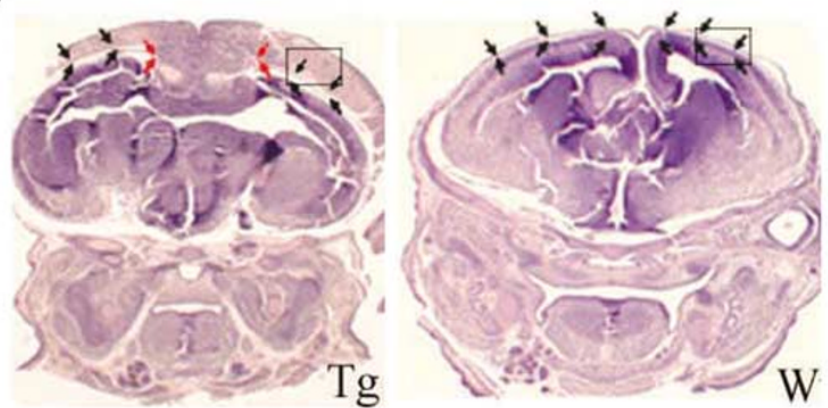

d
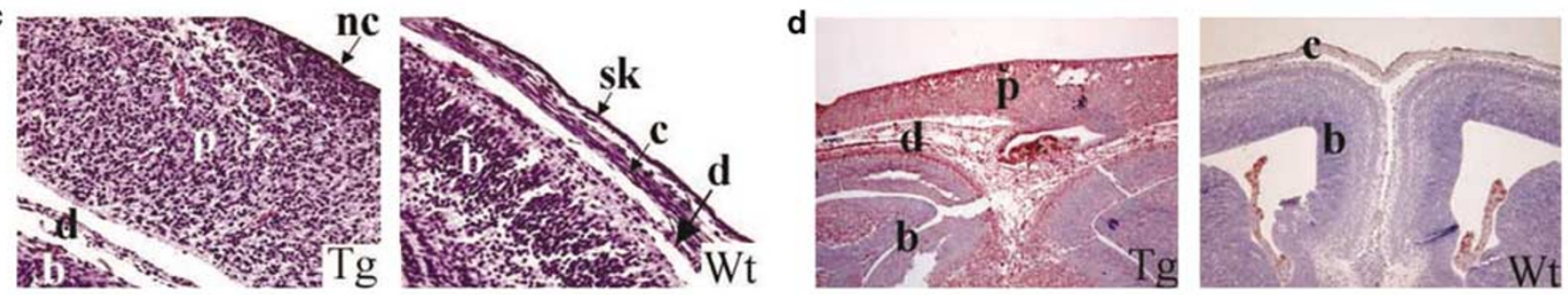

e
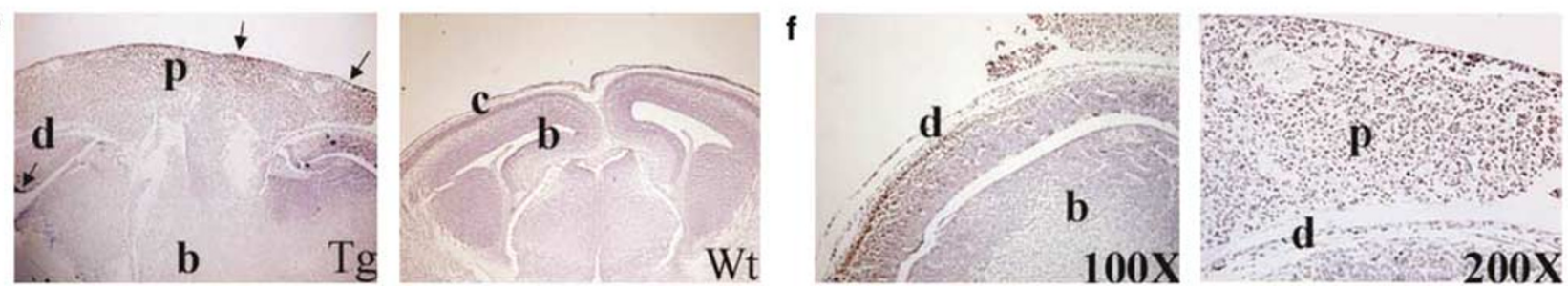

Figure 1 Acrania in non-viable E15.5 Nell-1 transgenic embryos. (a) Whole mount photo of an E15.5 Nell-1 transgenic mouse with acrania (left panel) and a wild-type littermate (right panel). Note the exencephaly-like phenotype as indicated by black arrows. (b) H\&E staining on a coronal section of a Nell-1 transgenic mouse (left panel) and wild-type littermate (right panel) head. Black arrows indicate the dura mater of transgenic mice, but the skin, calvaria, and dura mater of wild-type mice. Red arrows indicate the disrupted dura mater of transgenic mice. Areas within black boxes are magnified and further analyzed. (c) Highly magnified images of H\&E staining of the brain protruding from a transgenic (left panel) and normal brain and calvaria of wild type (right panel) mice head. (d) Immunohistochemistry detected intense Nell-1 production in the brain and dura mater of Nell-1 transgenic mice (left panel) and restricted and reduced staining in wild-type mice (right panel). (e) In situ TUNEL analysis of the protruding brain tissue from the Nell-1 transgenic mouse (left panel) and wild-type mouse (right panel) with apoptotic cells stained brown. Arrows point to representative apoptotic cells. (f) Magnified sections of in situ TUNEL staining on Nell-1 transgenic mouse sections. Original magnification: $\times 25$ (b), $\times 40(\mathbf{e})$, and $\times 100$ (d, f left panel), $\times 200$ (c, f right panel). Tg-transgenic; Wt-wild type; p-protruding brain; c-calvaria; b-brain; sk-skin, d-dura mater; nc-neural cells.

(Figure 2a) and moderately increased Fas-L (Figure 2b) production within the protruding brain and dura mater of Nell-1 transgenic mice as compared to the normal brain and calvaria of the wild-type littermates. The increase of both Fas and Fas-L within the protruding brain correlated to the increased apoptosis in this area as demonstrated by in situ TUNEL staining in Figure 1e. To further examine the mechanism of increased apoptosis in Nell-1 transgenic mice, apoptosis, Fas, and Fas-L production were analyzed at an earlier stage (E12.5). E12.5 Nell-1 transgenic embryos did not show a phenotype, yet did demonstrate increased Nell-1 production as compared to wild-type littermates (Figure 2c). Interestingly, E12.5 Nell-1 transgenic mice revealed dramatically increased Fas production as compared to wild-type mice (Figure 2d), but neither Fas-L nor
TUNEL staining demonstrated significant differences at this early time point (Figure 2e and f). The lack of significant apoptosis within Nell-1 transgenic mice may at least partially account for the lack of phenotype in these animals at this time. The mandible of Nell-1 transgenic mice was morphologically normal and the Meckel's cartilage did not display differential apoptosis rates as compared to wild-type littermates (data not shown).

\section{Increased Apoptosis Associated with Deformed Chondrocranium in E15.5 and Newborn Craniosynostotic Mice}

As previously reported, newborn Nell-1 transgenic mice show varied degrees of craniosynostosis (CS). ${ }^{28}$ 
a
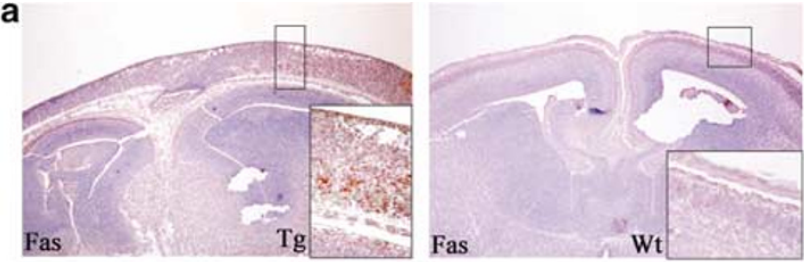

b
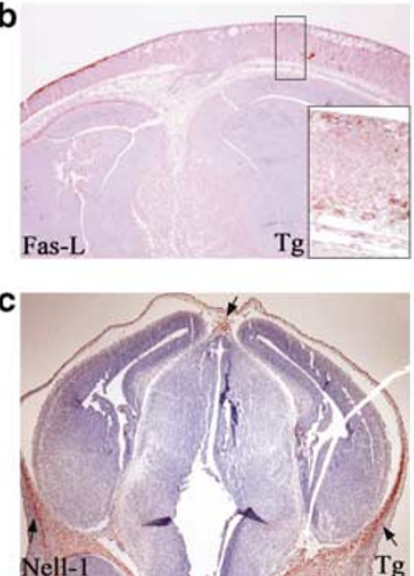
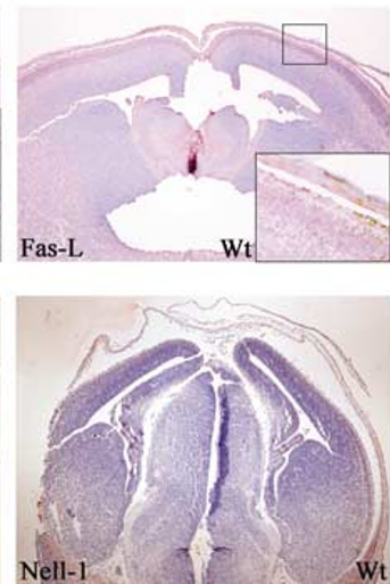

d

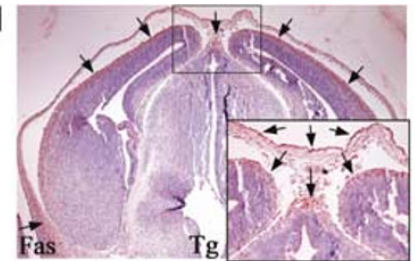

e

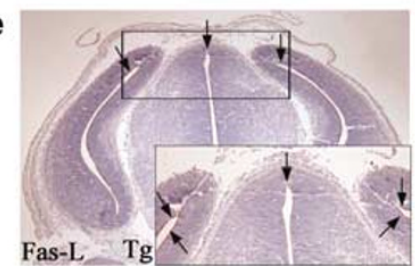

f

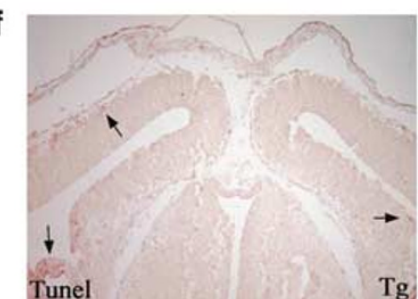

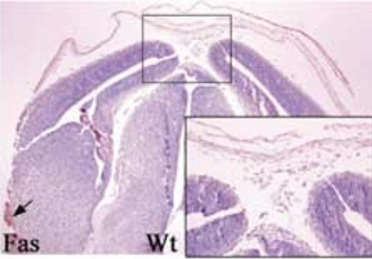
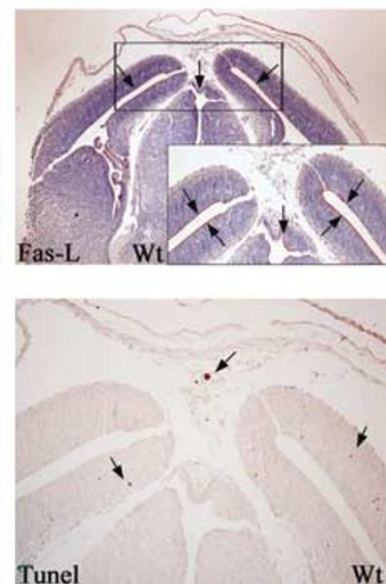

Figure 2 Fas and Fas-L production in E12.5 and E15.5 Nell-1 transgenic embryos. (a) Fas and (b) Fas-L immunostaining in the protruding brain and dura mater/calvaria of an E15.5 Nell-1 transgenic mouse embryo exhibiting acrania phenotype (left panel) as compared to a wild-type littermate (right panel). Representative areas are magnified in the lower right corner. (c) Nell-1 immunostaining in an E12.5 Nell-1 transgenic (left panel) and wild-type (right panel) mouse embryo. (d) Fas and (e) Fas-L immunostaining in an E12.5 Nell-1 transgenic (left panel) and wild-type (right panel) mouse embryo. Arrows point to areas of high intensity staining (red). Areas in black boxes are magnified in the lower right corner. (f) In situ TUNEL analysis in E12.5 Nell-1 transgenic mice (left panel) and wild-type (right panel) embryos. Black arrows point to red areas representing positive staining. Original magnification: $\times 40(\mathbf{a}-\mathbf{e}), \times 100(\mathbf{f}$ and lower right panel of $\mathbf{e}$ ), and $\times 200$ (lower right panels of $\mathbf{a}, \mathbf{b}$, and $\mathbf{d}$ ).

The data from the current study as well as other reported cases $^{22}$ described two newborn Nell-1 transgenic mice out of three litters $(2 / 19,10.53 \%)$ that survived to birth and displayed as encephalocele and a calvarial bone defect found in the occipital region (Figure 3a). In situ TUNEL identified increased apoptosis in the calvarial bone osteoblasts of these newborn Nell-1 transgenic mice as compared to wild-type littermates (Figure 3b). Coordinately, we also observed premature hypertrophy and apoptosis of chondrocytes throughout the distorted chondrocranium as compared to wild-type littermates (Figure 3c). The noted distortion of the chondrocranial morphology is similar to that seen in human cases of CS, such as those with Apert's and Crouzon's syndromes. ${ }^{33}$ It is possible that increased chondrocyte apoptosis in Nell-1 transgenic mice may relate to or contribute to malformation of the chondrocranium. Additionally, H\&E staining identified prematurely hypertrophic chondrocyte islands within the chondrocranium of E15.5 Nell-1 transgenic mouse embryos, but not in wild-type littermates (Figure 3d, left panel). In situ TUNEL staining revealed increased apoptosis within the chondrocranium of E15.5 Nell-1 transgenic mice (Figure 3d, right panel). Although chondrocytes in the chondrocranium will eventually hypertrophy, our data indicated that this event was exaggerated and occurred at an earlier stage in Nell-1 transgenic mice. Furthermore, Fas was highly produced in the cartilaginous chondrocranium of Nell-1 transgenic newborn mice as compared to wild-type littermates (Figure 3e), while Fas-L production was similar among the littermates (data not shown). Premature apoptosis and the appearance of empty lacunae with decreased matrix suggest a disruption in the pathway of endochondral bone formation in these Nell-1 transgenic mice. Both Nell-1 transgenic and wild-type littermates displayed equivalent levels of Fas production in Meckel's cartilage (data not shown).

\section{Increased Apoptosis of AdNell-1 Transduced Chondrocytes In Vitro}

Nell-1 transgenic mice displayed increased chondrocyte apoptosis in the distorted chondrocranium. Thus, we further characterized the role of Nell-1 in cultured chondrocytes (Figure $4 \mathrm{a}$ and b). With increased amounts of AdNell-1 transduction (50 and $200 \mathrm{pfu} / \mathrm{cell}$ ), cell cultures appeared more apoptotic with round and uplifted cells. To confirm the production of Nell-1 in these cultures, Western blot analysis revealed increased Nell-1 production upon transduction with 50 and 200 pfu/cell of AdNell-1 as opposed to untransduced or AdLacZ transduced chondrocytes (Figure 4c). In situ TUNEL 
a

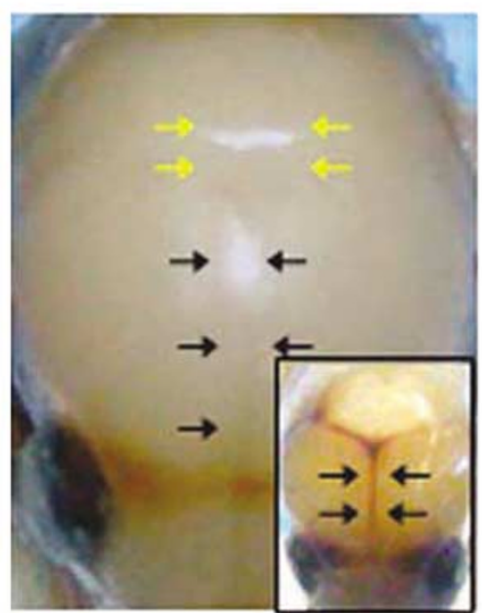

c

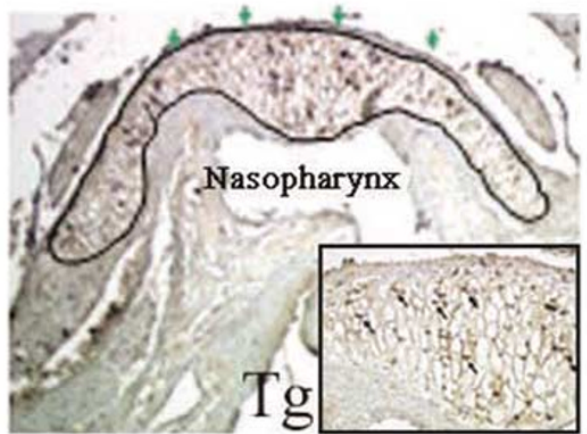

d

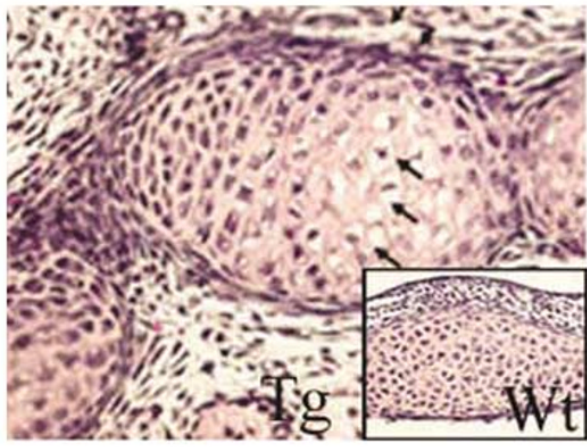

e

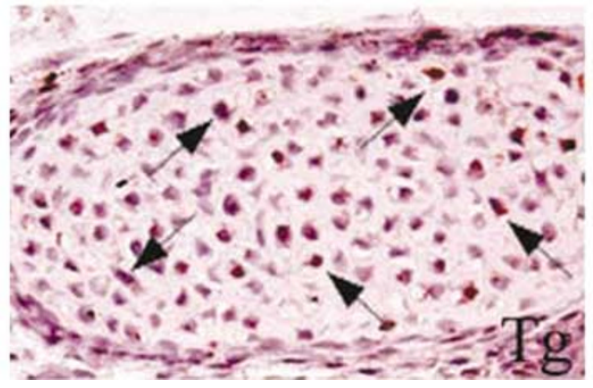

b
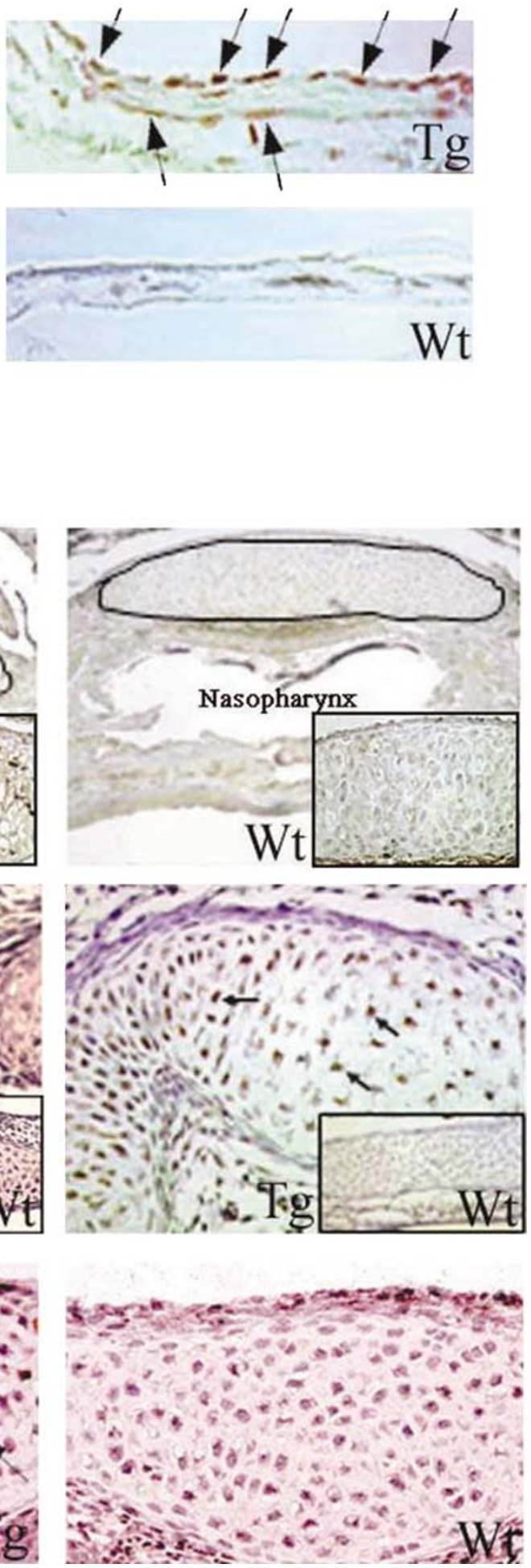

Figure 3 Apoptosis in newborn Nell-1 transgenic mice. (a) A newborn Nell-1 transgenic mouse whole head mount with wild-type head (lower right panel). Black arrows point to the posterior frontal and sagittal suture, while yellow arrows point to the protruding brain in the calvarial defect region. (b) In situ TUNEL analysis of a coronal section of newborn Nell-1 transgenic mouse calvarial bone (upper panel) and wild-type littermate calvaria (lower panel). Black arrows point to brown stained apoptotic osteoblast cells. (c) In situ TUNEL analysis of a coronal section of the chondrocranium region of the Nell-1 transgenic mouse (left panel) and wild-type mouse (right panel). Green arrows indicate the distorted chondrocranium (outlined by black line). Representative cartilaginous tissues with apoptotic chondrocytes are magnified in the lower right corner. (d) H\&E staining (left panel) and in situ TUNEL analysis (right panel) of Nell-1 transgenic and wild-type (lower right panels) E15.5 mouse chondrocranium. Black arrows point to hypertrophic chondrocytes or brown stained apoptotic cells. The TUNEL sections are counter stained with hematoxylin. (e) Fas immunostaining in Nell-1 transgenic (left panel) and wild-type (right panel) newborn littermate chondrocranium. Original magnification: $\times 40$ (c), $\times 200$ (b, d and lower panels of c and d), $\times 400(\mathbf{e})$. Tg-transgenic; Wt-wild-type. 

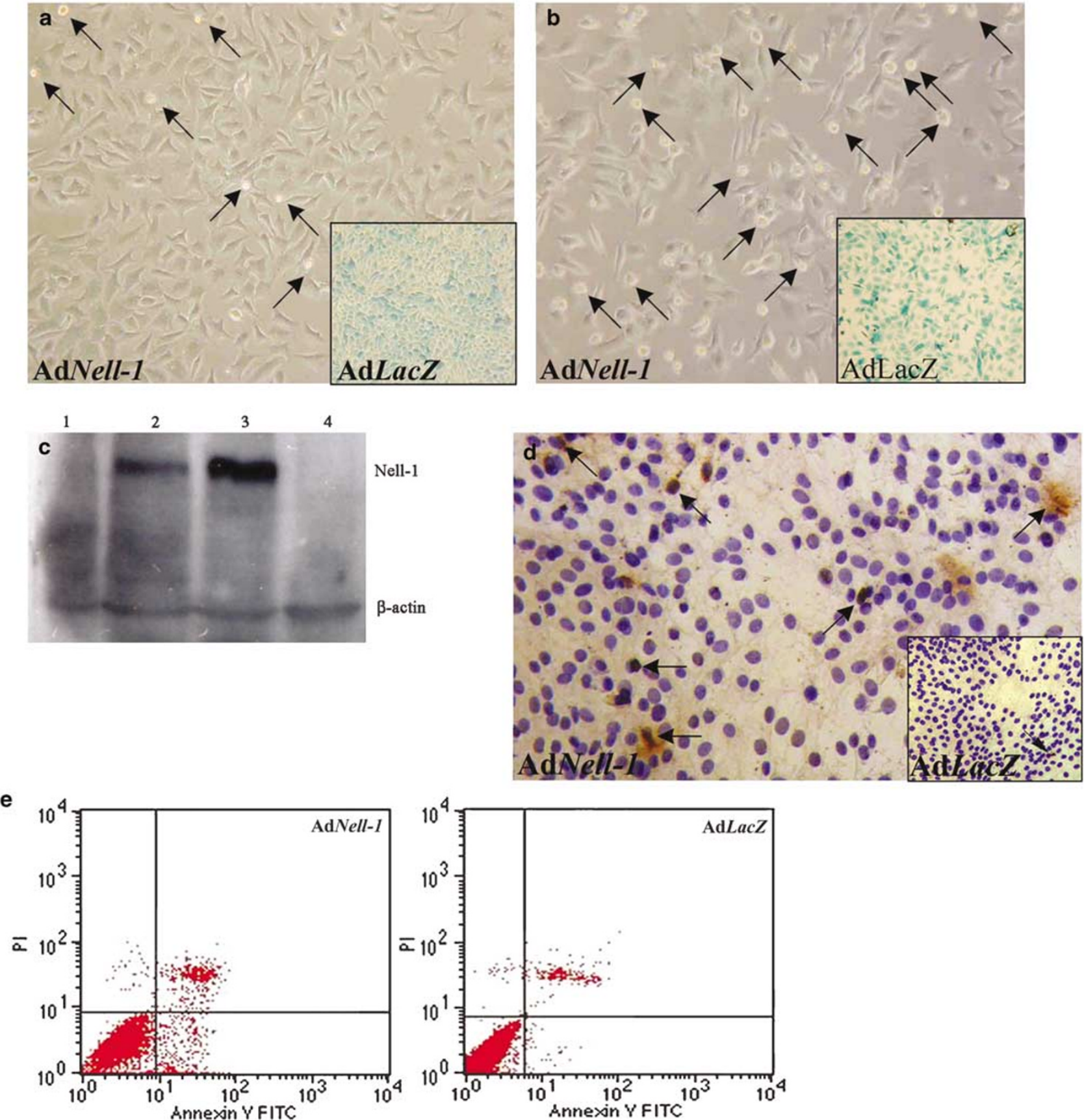

Figure 4 Apoptosis in cultured chondrocytes. White light image of cultured chondrocytes transduced with (a) 50 pfu/cell AdNell-1 or (b) $200 \mathrm{pfu} / \mathrm{cell}$ AdNell-1 and with AdLacZ control in the lower right corner, nine days after transduction. Black arrows point to apoptotic cells. (c) Western blot analysis of Nell-1 protein production in chondrocytes, three days after transduction with AdNell-1. Lane 1untransduced cells; lane 2-50 pfu/cell AdNell-1; lane 3-200 pfu/cell AdNell-1; lane 4-AdLacZ. (d) In situ TUNEL analysis of cultured chondrocytes, nine days after transduction. (e) Flow cytometry of cultured chondrocytes transduced with AdNell-1 (left panel) or AdLacZ (right panel), six days after transduction. Cells are labeled with Annexin V and propidium iodide (PI) for analysis. Note the significantly $(P<0.001)$ increased number of apoptotic cell stained with high levels of Annexin V or Annexin V/PI and found in the lower and upper right quadrant of AdNell-1, as opposed to AdLacZ, transduced cells.

analysis demonstrated that cultured chondrocytes displayed increased apoptosis at day 9 after transduction with AdNell-1 when compared to control AdLacZ (Figure 4d). Additionally, increasing amounts of AdNell-1 transduction and Nell-1 protein production resulted in elevated cell death rates.
Flow cytometry further confirmed a significantly increased apoptotic rate in AdNell-1 as opposed to AdLacZ transduced chondrocytes (Figure 4e), even at day 6 post-transduction. These results further verified the in vivo findings of Nell-1 overexpression induced apoptosis in chondrocytes. 


\section{Discussion}

Neural tube defects arise from a variety of heterogeneous mechanisms. ${ }^{34,35}$ For this reason, NTDs can be secondary to defects occurring in non-neural developmental systems. ${ }^{6}$ We have previously identified exencephaly-like characteristics in Nell-1 transgenic embryos. ${ }^{22,28}$ Our previous studies have demonstrated the role of NELL-1 in human and murine CS, ${ }^{22,23,28}$ while the current studies were performed to further investigate the effects of Nell-1 overexpression on mouse embryonic development and the corresponding molecular mechanism.

In the current study, additional Nell-1 transgenic mice were examined due to the presence of NTDs found at E15.5. In addition to two litters previously reported, we examined two additional litters of E15.5 embryos as well as E12.5 mouse embryos from timed-mated Nell-1 transgenic F1 mice with wildtype littermates. A total of three non-viable exencephaly-like mouse embryos out of 16 Nell-1 transgenic E15.5 embryos (3/16, 18.75\%) were identified. It has been suggested that the penetrance of NTDs can be affected at the genetic level and likely modified by environmental factors such as dietary intake. ${ }^{36}$ This finding points to the complexity and heterogeneity of the pathogenesis of NTDs, which involves the regulation of NTD-associated genes and metabolic pathways. The phenotypes of transgenic mice at an earlier stage (E12.5) were indistinguishable and had normal appearances. A histological analysis of the abnormal E15.5 transgenic mice embryos showed features of a postneurulation NTD called acrania, characterized by a complete absence of the calvarial bone and abnormal brain development. It differed from primary acalvaria, in which the absence of calvarial bone, dura mater, and intact overlying skin are the hallmarks of pathological changes. Furthermore, it also differed from open NTD exencephaly, with which acrania was frequently accompanied. Practically, it is difficult to distinguish exencephaly from acrania morphologically, because sometimes both can show partial or complete absence of membranous flat bones of the neurocranium with abnormal brain development and commonly intact skull base. However, mechanistically, exencephaly usually occurs at early embryonic stage prior to the closure of neural tube, while acrania is a postneurulation NTDs. ${ }^{3}$ Our current data strongly suggested that overexpression of Nell-1 could induce the acrania-like phenotype in mouse embryos at relatively late gestation stage; making Nell-1 a novel NTD-associated molecule.

Previously, we reported that a Nell-1 transgenic mouse model, exhibiting generalized Nell-1 overexpression, resulted in increased osteoblast differentiation, followed by an increase in osteoblast apoptosis. ${ }^{22}$ In that model, Nell-1 exerted its apoptotic effects on differentiated osteoblasts rather than undifferentiated mesenchymal cells. Consequently, the upregulation of Nell-1 in the calvaria did not decrease the osteogenic population, but instead enhanced the natural progression of osteoblast differentiation leading to increased bone formation. The enhanced differentiation may also play an important role in cranial suture closure or CS. Those findings suggested that apoptosis plays a role in altered calvarial bone formation. ${ }^{22}$

Neural cells/neuroblasts within the protruding brain of Nell-1 transgenic mice demonstrated massive apoptosis as compared to the normal brain of wild-type littermates. The process of apoptosis has been suggested to be tightly linked to cell divi$\operatorname{sion}^{37,38}$ both representing a time when neurons are unable to migrate within the brain. ${ }^{39}$ Thus, it is possible that the inability of the apoptotic neural cells to migrate, may have contributed to acrania in this model, as seen in other models. ${ }^{19,40-42}$ Specifically, Msx2 transgenic mice display increased death of CNC cells, resulting in a cell number deficiency in branchial arches associated with craniofacial morphogenesis. ${ }^{41}$ Interestingly, Msx2 induces apoptosis in CNC cells of rhombomeres 3 and $5,{ }^{43}$ but increases proliferation of osteoblasts in the osteogenic front of postnatal mice, ${ }^{44}$ leading to exencephaly-like NTDs. This observation is recapitulated in Tcof1 heterozygous mice and Tulp3 transgenic mice, which display increased neuroepithelial apoptosis during development, exencephaly, and premature death. ${ }^{19,42}$ Thus, the various allele deficiencies result in similar phenotypes. An evaluation of the signaling pathways in the various transgenic animals could possibly identify similar mechanisms of action, leading us closer to preventative measures in humans. The data suggest that the mechanism of Nell-1 overexpression-induced acrania may be the coordinated induction of apoptosis in several cell types, a finding also reported in $M s \times 2$ transgenic mice. ${ }^{41}$

Nell-1 has been previously reported to signal through the PKC pathway, ${ }^{25}$ which is known to induce apoptosis in osteoblasts. ${ }^{45}$ In fact, in other systems, PKC activation has also induced FasL gene transcription. ${ }^{46}$ A predominant pathway of apoptosis in calvarial osteoblasts. ${ }^{20,21,47,48}$ and brain ${ }^{31,49-51}$ is the Fas mediated pathway. In this pathway, Fas-L activates the Fas receptor to trigger several signaling cascades including Fas-associated death domain (FADD) protein, receptor interacting protein (RIP), or Fas death domain-associated protein (DAXX) and eventually leading to cell death ${ }^{52-56}$ Previously, we have reported an upregulation of Fas antigen by overexpression of Nell-1 in MC3T3-E1 osteoblasts in culture. ${ }^{22}$ Currently, we present the in vivo upregulation of Fas and/or Fas-L within the calvaria, protruded brain, and chondrocranium of E12.5 and E15.5 Nell-1 transgenic mice. The occurrence of Fas upregulation was prior to the onset of apoptosis and phenotypic changes, suggesting that increased Fas signaling was one of the possible mechanisms responsible for increased apoptosis and thus the NTDs in these mice. 
An interesting finding of our study was that Nell-1 transgenic mice exhibited common severe craniofacial anomalies also observed in humans, including both acrania and encephalocele. Interestingly, the pathology of both phenotypes involves apoptosis of calvaria, brain, and cartilage. It is possible that they are caused by different mechanisms; acrania may be induced by apoptosis and CNC differentiation defects, while encephalocele may be caused by mechanical factors that increase intracranial pressure and push the brain tissue to herniate. The data suggest that while a moderate upregulation of Nell-1 expression leads to accelerated osteoblast differentiation and craniosynostosis, an exaggerated overexpression of Nell-1, such as in the pathologic conditions observed in several Nell-1 overexpression mice, induced increased apoptosis and resulted in NTDs such as acrania (Figure 5). However, this is a highly simplified mechanistic hypothesis of NTDs involving Nell-1 overexpression. It emphasizes that Nell-1-induced acrania may be due to either the enhanced apoptosis that leads to a decrease in mineralization, a disruption in cell migration, or a combination of these mechanisms. Additionally, Nell-1 expression is upregulated by FGF-2 and TGF- $\beta 1$ signaling (unpublished data), also known to affect suture biology. Although we have no data relating exact copy number to the level of transgene expression, the forced expression of Nell-1 on CNC cells during development induced NTDs and thus demonstrated Nell-1's pharmacological effect. These data suggest that increased severity of the phenotype is closely associated with increased expression of Nell-1.

Another interesting observation in transgenic E15.5 embryos and newborns was the significant number of apoptotic chondrocytes in the chondrocranium that also appeared hypertrophic. The origin of cells within the chondrocranium varies, with the neurocranium (surrounding the brain) of mixed mesenchymal and neural crest origin and the viscerocranium (forming facial bones) of neural crest origin. ${ }^{57,58}$ Although Nell-1 is preferentially expressed by CNC cells, it is not exclusively expressed by these cells. Additionally, the cellular targets of Nell-1 have not been elucidated due to the fact that the Nell-1 receptor has not been identified.
The resulting malformed chondrocranium was analogous to that observed in Apert's and Crouzon's syndromes, ${ }^{33}$ including spheno-occipital and petrooccipital synchondrosis. ${ }^{59}$ This result was not reported in our previous publication, because of the inability of microCT scans to detect unmineralized cartilage. ${ }^{28}$ In the above mentioned syndromes, craniofacial pathogenesis may have had primitive alterations of the cartilaginous template from which the endochondral bones are derived. The premature fusion of the synchondrosis of the cranial base causes distortion development, suggesting mechanical effect. Interestingly, the current data suggests a cellular etiology.

Nell-1's ability to induce apoptosis in chondrocytes was further verified by increased apoptosis of cultured chondrocytes transduced with a high dose of AdNell-1. Like the in vivo findings, the transduced chondrocytes increased apoptosis as compared to AdLacZ transduced cells. Also, the subcutaneous implantation of scaffold-supported chondrocytes transduced with AdNell-1 can produce a bone-like tissue mass consisting of highly hypertrophic chondrocytes in comparison with AdLacZ control (unpublished data). This further indicated that Nell-1 plays important roles on the differentiation or maturation of chondrocytes.

A debate continues about whether the cranial base deformities observed in these CS syndromes are primary or secondary to suture fusion. Virchow believed that the cranial base malformation is secondary to constraints resulting from premature suture closure. ${ }^{60}$ However, recent evidence suggests that the cartilaginous cranial base is primarily involved. ${ }^{61}$ Additionally, the current data suggests an association between Nell-1 overexpression-induced apoptosis and the malformation of the cranial base, although the data does not directly prove a cause and effect relationship. This suggests that the deformed cranial base resulted from the cellular mechanisms of apoptosis, mechanical compression, or most likely both.

Although the upregulation of Nell-1 expression affected the pathology of craniofacial development, it did not have a significant effect on mandibular development. This result was unexpected as cells within the mandible originate both from CNC and

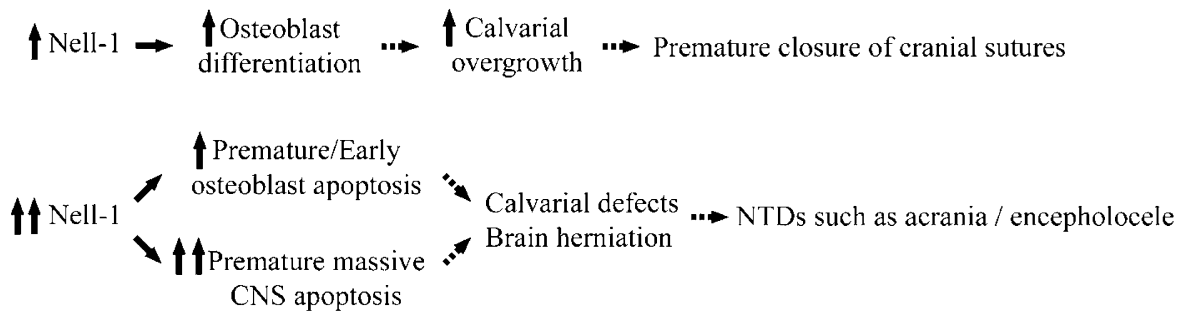

Figure 5 Hypothetical model of Nell-1 signaling in craniofacial deformities. Moderate upregulation of Nell-1 induces osteoblast differentiation. This results in calvarial bone overgrowth and consequently premature suture closure. Exaggerated Nell-1 overexpression in certain stages of embryonic development induces premature/early osteoblast apoptosis and therefore reduces the number of osteoblasts forming bone. Defects in calvarial bone formation result in craniofacial anomalies such as acrania/ exencephaly or encephalocele. Dashed lines represent potential pathways of modulation. 
non-CNC derived cells. ${ }^{62}$ Similar findings have also been demonstrated in an AP-2 deficient mouse model where most achordal bone formation (of CNC origin) was deformed and other bones of CNC origin, such as the hyoid, Meckel's cartilage, and mandibles, were indistinguishable from wild-type ${ }^{63}$ In normal mandibular development, apoptosis in the bone and Meckel's cartilage of the mandible occurs through the Fas-Fas-L pathway. ${ }^{64}$ Pathologically, Meckel's cartilage is formed early in development ${ }^{65,66}$ and is ultimately degraded and replaced by intramembranous bone, ${ }^{67}$ acting as a frame for mandibular bone formation. ${ }^{68}$ Although chondrocytes located at the posterior end of Meckel's cartilage do ossify to form the malleus and incus bones of the inner ear, ${ }^{69}$ chondrocytes within the body of Meckel's cartilage do not express type X collagen, mineralize, or form bone. ${ }^{70,71}$ In Nell-1 transgenic mice, the number of apoptotic cells within the Meckel's cartilage was equivalent to that in wild-type mice (data not shown), thus the anatomy of this bone was not altered. Although the underlying reason is unknown, we speculate that the normal occurrence of apoptosis in this tissue could not be further affected by the increased presence of Nell-1, due to the non-osteogenic fate of cells within Meckel's cartilage and the fact that Nell-1's effects are restricted to osteochondral bone formation.

In conclusion, our results suggest that Nell-1 overexpression induced dysregulation of CNS and CNC cells during cranial development. These effects resulted in craniofacial developmental deformities including acrania at relatively late embryonic stage E15.5 and encephalocele in addition to craniosynostosis in neonatal stage, which may be related to massive apoptosis of osteoblasts, neural cells, and chondrocytes through the Fas signaling pathway. These developmental defects demonstrate multifactorial inheritance patterns in human populations, suggesting a complex interplay of genetic factors, which may be targeted by the Nell-1 protein or more complicated networks involving other NTD-associated molecules and contributions from epigenetic factors. Thus far, it remains unknown as to which mouse gene homologues plays a key role in the etiology of human NTDs, as no convincing definition of a predisposing allele involved in more than one individual NTD case in humans has been identified. ${ }^{72}$ Further elucidation of the Nell-1 signaling cascade may provide insight into the mechanism of NTD formation as well as other craniofacial anomalies in humans.

\section{Acknowledgements}

This research is supported by the Wunderman Family Foundation, March of Dimes Birth Defect Foundation \#6-FY02-163, NIH/NIDCR RO3 DE 014649-01, NIH/NIDCR K23DE00422, NIH
DE016107-01, the Thomas R Bales endowed Chair, and NIH T32 UCLA Research Training Grant. We thank the Jane and Jerry Weintraub Center for Reconstructive Biotechnology, UCLA for technical support.

\section{Duality of interest}

Dr Kang Ting and Dr Xinli Zhang are co-founders of Bone Biologics Inc., which owns the license on the Nell-1 patent from UCLA.

\section{References}

1 Matsumoto A, Hatta T, Moriyama K, et al. Sequential observations of exencephaly and subsequent morphological changes by mouse exo utero development system: analysis of the mechanism of transformation from exencephaly to anencephaly. Anat Embryol (Berlin) 2002;205:7-18.

2 Laurence KM. Principles and practice of medical genetics. In: Emery AEH, Rimoin DL (eds). Churchill, Livingston, Edenburgh, 1990, pp 323-346.

3 Lemire RJ. Neural tube defects. JAMA 1988;259: 558-562.

4 Lomaga MA, Henderson JT, Elia AJ, et al. Tumor necrosis factor receptor-associated factor 6 (TRAF6) deficiency results in exencephaly and is required for apoptosis within the developing CNS. J Neurosci 2000;20:7384-7393.

5 Greene ND, Copp AJ. Mouse models of neural tube defects: investigating preventive mechanisms. Am J Med Genet C Semin Med Genet 2005;135:31-41.

6 Harris MJ, Juriloff DM. Mini-review: toward understanding mechanisms of genetic neural tube defects in mice. Teratology 1999;60:292-305.

7 Harris CP, Townsend JJ, Carey JC. Acalvaria: a unique congenital anomaly. Am J Med Genet 1993;46:694-699.

8 Ekici E, Gulmezoglu AM. Sonographic diagnosis of fetal acrania. J Clin Ultrasound 1991;19:363-366.

9 Vergani P, Ghidini A, Sirtori M, et al. Antenatal diagnosis of fetal acrania. J Ultrasound Med 1987;6: 715-717.

10 Couly GF, Coltey PM, Le Douarin NM. The triple origin of skull in higher vertebrates: a study in quail-chick chimeras. Development 1993;117:409-429.

11 Chandran S, Lim MK, Yu VY. Fetal acalvaria with amniotic band syndrome. Arch Dis Child Fetal Neonatal Ed 2000;82:F11-F13.

12 Horstadius S. The mechanics of sea urchin development. Annee Biol 1950;26:381-398.

13 Poelmann RE. Differential mitosis and degeneration patterns in relation to the alterations in the shape of the embryonic ectoderm of early post-implantation mouse embryos. J Embryol Exp Morphol 1980;55: $33-51$.

14 Serbedzija GN, Bronner-Fraser M, Fraser SE. Vital dye analysis of cranial neural crest cell migration in the mouse embryo. Development 1992;116:297-307.

15 Noden DM. An analysis of migratory behavior of avian cephalic neural crest cells. Dev Biol 1975;42:106-130.

16 Tosney KW. The segregation and early migration of cranial neural crest cells in the avian embryo. Dev Biol 1982;89:13-24. 
17 Chai $\mathrm{Y}$, Jiang $\mathrm{X}$, Ito $\mathrm{Y}$, et al. Fate of the mammalian cranial neural crest during tooth and mandibular morphogenesis. Development 2000;127:1671-1679.

18 Horiuchi K, Ariga T, Fujioka H, et al. Treacher Collins syndrome with craniosynostosis, choanal atresia, and esophageal regurgitation caused by a novel nonsense mutation in TCOF1. Am J Med Genet 2004;128A: 173-175.

19 Dixon J, Brakebusch C, Fassler R, et al. Increased levels of apoptosis in the prefusion neural folds underlie the craniofacial disorder, Treacher Collins syndrome. Hum Mol Genet 2000;9:1473-1480.

20 Tsuboi M, Kawakami A, Nakashima T, et al. Tumor necrosis factor-alpha and interleukin-1beta increase the Fas-mediated apoptosis of human osteoblasts. J Lab Clin Med 1999;134:222-231.

21 Kawakami A, Eguchi K, Matsuoka N, et al. Fas and Fas ligand interaction is necessary for human osteoblast apoptosis. J Bone Miner Res 1997;12:1637-1646.

22 Zhang X, Carpenter D, Bokui N, et al. Overexpression of Nell-1, a craniosynostosis-associated gene, induces apoptosis in osteoblasts during craniofacial development. J Bone Miner Res 2003;18:2126-2134.

23 Ting K, Vastardis H, Mulliken JB, et al. Human NELL-1 expressed in unilateral coronal synostosis. J Bone Miner Res 1999;14:80-89.

24 Watanabe TK. Cloning and characterization of two novel human cDNAs (NELL1 and NELL2) encoding proteins with six EGF-like repeats. Genomics 1996; 38:273-276.

25 Kuroda S, Tanizawa K. Involvement of epidermal growth factor-like domain of NELL proteins in the novel protein-protein interaction with protein kinase C. Biochem Biophys Res Commun 1999;265:752-757.

26 Kuroda S, Oyasu M, Kawakami M, et al. Biochemical characterization and expression analysis of neural thrombospondin-1-like proteins NELL1 and NELL2. Biochem Biophys Res Commun 1999;265:79-86.

27 Bornstein P, McKinney CE, LaMarca ME, et al. Metaxin, a gene contiguous to both thrombospondin 3 and glucocerebrosidase, is required for embryonic development in the mouse: implications for Gaucher disease. Proc Natl Acad Sci USA 1995;92:4547-4551.

28 Zhang X, Kuroda S, Carpenter D, et al. Craniosynostosis in transgenic mice overexpressing Nell-1. J Clin Invest 2002;110:861-870.

29 Lotz M, Hashimoto S, Kuhn K. Mechanisms of chondrocyte apoptosis. Osteoarthritis Cartilage 1999;7:389-391.

30 Cheema ZF, Santillano DR, Wade SB, et al. The extracellular matrix, p53 and estrogen compete to regulate cell-surface Fas/Apo-1 suicide receptor expression in proliferating embryonic cerebral cortical precursors, and reciprocally, Fas-ligand modifies estrogen control of cell-cycle proteins. BMC Neurosci 2004;5:11.

31 Cheema ZF, Wade SB, Sata M, et al. Fas/Apo [apoptosis]-1 and associated proteins in the differentiating cerebral cortex: induction of caspase-dependent cell death and activation of NF-kappaB. J Neurosci 1999;19:1754-1770.

32 Sayah DN, Soo C, Shaw WW, et al. Downregulation of apoptosis-related genes in keloid tissues. J Surg Res 1999;87:209-216.

33 Cohen SR, Holmes RE, Amis P, et al. Internal craniofacial distraction with biodegradable devices: early stabilization and protected bone regeneration. J Craniofac Surg 2000;11:354-366.
34 Inoue $\mathrm{T}$, Hatayama M, Tohmonda T, et al. Mouse Zic5 deficiency results in neural tube defects and hypoplasia of cephalic neural crest derivatives. Dev Biol 2004;270:146-162.

35 Hunt JA, Hobar PC. Common craniofacial anomalies: facial clefts and encephaloceles. Plast Reconstr Surg 2003;112:606-615; quiz 616, 722.

36 Juriloff DM, Harris MJ. Mouse models for neural tube closure defects. Hum Mol Genet 2000;9:993-1000.

37 Evan GI, Brown L, Whyte M, et al. Apoptosis and the cell cycle. Curr Opin Cell Biol 1995;7:825-834.

38 Jacobson MD, Weil M, Raff MC. Programmed cell death in animal development. Cell 1997;88:347-354.

39 Li S, Qiu F, Xu A, et al. Barhl1 regulates migration and survival of cerebellar granule cells by controlling expression of the neurotrophin-3 gene. J Neurosci 2004;24:3104-3114.

40 Liu A, Losos K, Joyner AL. FGF8 can activate Gbx2 and transform regions of the rostral mouse brain into a hindbrain fate. Development 1999;126:4827-4838.

41 Winograd J, Reilly MP, Roe R, et al. Perinatal lethality and multiple craniofacial malformations in MSX2 transgenic mice. Hum Mol Genet 1997;6:369-379.

42 Ikeda A, Ikeda S, Gridley T, et al. Neural tube defects and neuroepithelial cell death in Tulp3 knockout mice. Hum Mol Genet 2001;10:1325-1334.

43 Ellies DL, Church V, Francis-West P, et al. The WNT antagonist cSFRP2 modulates programmed cell death in the developing hindbrain. Development 2000;127: 5285-5295.

44 Liu YH, Tang Z, Kundu RK, et al. Msx2 gene dosage influences the number of proliferative osteogenic cells in growth centers of the developing murine skull: a possible mechanism for MSX2-mediated craniosynostosis in humans. Dev Biol 1999;205:260-274.

45 Hay E, Lemonnier J, Fromigue O, et al. Bone morphogenetic protein-2 promotes osteoblast apoptosis through a Smad-independent, protein kinase Cdependent signaling pathway. J Biol Chem 2001;276: 29028-29036.

46 Pardo J, Buferne M, Martinez-Lorenzo MJ, et al. Differential implication of protein kinase $\mathrm{C}$ isoforms in cytotoxic $\mathrm{T}$ lymphocyte degranulation and TCRinduced Fas ligand expression. Int Immunol 2003; 15:1441-1450.

47 Ozeki N, Mogi M, Nakamura H, et al. Differential expression of the Fas-Fas ligand system on cytokineinduced apoptotic cell death in mouse osteoblastic cells. Arch Oral Biol 2002;47:511-517.

48 Lemonnier J, Hay E, Delannoy $\mathrm{P}$, et al. Increased osteoblast apoptosis in apert craniosynostosis: role of protein kinase $\mathrm{C}$ and interleukin-1. Am J Pathol 2001;158:1833-1842.

49 French LE, Tschopp J. Constitutive Fas ligand expression in several non-lymphoid mouse tissues: implications for immune-protection and cell turnover. Behring Inst Mitt 1996;97:156-160.

50 Nishimura T, Akiyama $\mathrm{H}$, Yonehara S, et al. Fas antigen expression in brains of patients with Alzheimer-type dementia. Brain Res 1995;695:137-145.

51 Voiculescu B, Nat R, Lin E, et al. Apoptosis in human embryo development: 1. Cerebral cortex. J Cell Mol Med 2000;4:284-288.

52 Suda T, Takahashi T, Golstein P, et al. Molecular cloning and expression of the Fas ligand, a novel member of the tumor necrosis factor family. Cell 1993;75:1169-1178. 
53 Friesen C, Herr I, Krammer PH, et al. Involvement of the CD95 (APO-1/FAS) receptor/ligand system in druginduced apoptosis in leukemia cells. Nat Med 1996;2:574-577.

54 Boldin MP, Varfolomeev EE, Pancer Z, et al. A novel protein that interacts with the death domain of Fas/ APO1 contains a sequence motif related to the death domain. J Biol Chem 1995;270:7795-7798.

55 Chinnaiyan AM, O’Rourke K, Tewari M, et al. FADD, a novel death domain-containing protein, interacts with the death domain of Fas and initiates apoptosis. Cell 1995;81:505-512.

56 Chinnaiyan AM, Tepper CG, Seldin MF, et al. FADD/ MORT1 is a common mediator of CD95 (Fas/APO-1) and tumor necrosis factor receptor-induced apoptosis. J Biol Chem 1996;271:4961-4965.

57 Lemire RJ. Embryology of the skull. In: Cohen MM, MacLean RE (eds). Craniosynostosis: Diagnosis, Evaluation, and Management. Oxford University Press: New York, Oxford, 2000, pp 24-34.

58 Mooney MP, Siegel MI, Smith TD, et al. Evolutionary changes in the cranial vault and base: establishing the primate form. In: Mooney MP, Siegel MI (eds). Understanding Craniofacial Anomalies, The Etiopathogenesis of Craniosynostoses and Facial Clefting. WileyLiss, Inc.: New York, 2002, pp 275-294.

59 Cohen MM, MacLean RE. Apert syndrome. In: Cohen MM, MacLean RE (eds). Craniosynostosis, Diagnosis, Evaluation, and Management. Oxford University Press: Oxford, 2000, pp 316-353.

60 Virchow $H$. Ueber den Cretinismus, namentlich in Franken, und ueber pathologische Schaedelforamen. Verh Phys Med Ges Wuerzburg 1852;2:230-271.

61 Avantaggiato A, Carinci F, Curioni C. Apert's syndrome: cephalometric evaluation and considerations on pathogenesis. J Craniofac Surg 1996;7:23-31.

62 Nichols DH. Neural crest formation in the head of the mouse embryo as observed using a new histo logical technique. J Embryol Exp Morphol 1981;64: 105-120.

63 Zhang J, Hagopian-Donaldson S, Serbedzija G, et al. Neural tube, skeletal and body wall defects in mice lacking transcription factor AP-2. Nature 1996;381: 238-241.

64 Hatakeyama S, Tomichi N, Ohara-Nemoto Y, et al. The immunohistochemical localization of Fas and Fas ligand in jaw bone and tooth germ of human fetuses. Calcif Tissue Int 2000;66:330-337.

65 Radlanski RJ, Renz H, Klarkowski MC. Prenatal development of the human mandible. 3D reconstructions, morphometry and bone remodelling pattern, sizes 12-117 mm CRL. Anat Embryol (Berlin) 2003; 207:221-232.

66 Schneider RA, Helms JA. The cellular and molecular origins of beak morphology. Science 2003;299: 565-568.

67 Frommer J, Margolies MR. Contribution of Meckel's cartilage to ossification of the mandible in mice. J Dent Res 1971;50:1260-1267.

68 Sperber GH. Craniofacial Development. B.C. Decker: London, 2001.

69 Richany SF, Bast TH, Anson BJ. The development of the first branchial arch in man and the fate of Meckel's cartilage. Q Bull Northwest Univ Med Sch 1956;30:331-355.

70 Chung KS, Park HH, Ting K, et al. Modulated expression of type X collagen in Meckel's cartilage with different developmental fates. Dev Biol 1995; 170:387-396.

71 Bhaskar SN, Weinmann JP, Schour I. Role of Meckel's cartilage in the development and growth of the rat mandible. J Dent Res 1953;32:398-410.

72 Stegmann K, Boecker J, Richter B, et al. A screen for mutations in human homologues of mice exencephaly genes Tfap2alpha and Msx2 in patients with neural tube defects. Teratology 2001;63:167-175. 